\title{
Aberrant hypomethylation-mediated CD147 overexpression promotes aggressive tumor progression in human prostate cancer
}

\author{
YU-XIANG LIANG $^{1 *}$, RU-JUN MO ${ }^{1 *}$, HUI-CHAN HE ${ }^{1 *}$, JIA-HONG CHEN $^{1}$, JUN ZOU $^{2}$, \\ ZHAO-DONG HAN ${ }^{1}$, JIAN-MING LU ${ }^{1}$, CHAO CAI ${ }^{1}$, YAN-RU ZENG ${ }^{1}$, \\ WEI-DE ZHONG $^{1,3}$ and CHIN-LEE WU ${ }^{4,5}$
}

\author{
${ }^{1}$ Department of Urology, Guangdong Key Laboratory of Clinical Molecular Medicine and Diagnostics, \\ Guangzhou First People's Hospital, Guangzhou Medical University, Guangzhou, Guangdong 510180; ${ }^{2}$ Department of Urology, \\ The Third Affiliated Hospital of Guangzhou Medical University, Guangzhou Medical University, \\ Guangzhou, Guangdong 510180; ${ }^{3}$ Guangdong Provincial Institute of Nephrology, \\ Southern Medical University, Guangzhou, Guangdong 510515, P.R. China; \\ Departments of ${ }^{4}$ Pathology and ${ }^{5}$ Urology, Massachusetts General Hospital \\ and Harvard Medical School, Boston, MA 02114, USA
}

Received November 19, 2014; Accepted March 5, 2015

DOI: $10.3892 /$ or.2015.3870

\begin{abstract}
Our previous study revealed the potential role of CD147 in human prostate cancer (PCa). Here, we investigated the CD147 promoter methylation status and the correlation with tumorigenicity in human PCa. CD147 mRNA and protein expression levels were both significantly higher in the 4 PCa cell lines, than in the 2 non-tumorigenic benign human prostatic epithelial cell lines (all $\mathrm{P}<0.01$ ). We showed hypomethylation of promoter regions of CD147 in PCa cell lines with significant CD147 expression as compared to non-tumorigenic benign human prostatic epithelial cell lines slowly expressing CD147. Additionally, the treatment of methylated cell lines with 5-aza-2'-deoxycytidine increased CD147 expression significantly in low-expressing cell lines and also activated the expression of matrix metalloproteinase (MMP)-2, which may be one of the most important downstream targets of CD147. Furthermore, PCa tissues displayed decreased DNA methylation in the promoter region of CD147 compared to the corresponding non-cancerous prostate tissues, and methylation intensity correlated inversely with the CD147 mRNA levels. There was a significant negative correlation between CD147 mRNA levels and the number of
\end{abstract}

Correspondence to: Professor Wei-De Zhong, Department of Urology, Guangdong Key Laboratory of Clinical Molecular Medicine and Diagnostics, Guangzhou First People's Hospital, Guangzhou Medical University, Guangzhou, Guangdong 510180, P.R. China

E-mail: zhongwd2009@live.cn

${ }^{*}$ Contributed equally

Key words: prostate cancer, tumor progression, tumorigenesis, CD147, DNA methylation methylated sites in $\mathrm{PCa}$ tissues $(\mathrm{r}=-0.467, \mathrm{P}<0.01)$. In conclusion, our data offer convincing evidence for the first time that the DNA promoter hypomethylation of CD147 may be one of the regulatory mechanisms involved in the cancer-related overexpression of CD147 and may play a crucial role in the tumorigenesis of $\mathrm{PCa}$.

\section{Introduction}

Prostate cancer ( $\mathrm{PCa})$ is the most common solid cancer and the second leading cause of cancer-related deaths in men (1). It is a clinically heterogeneous-multifocal disease and the incidents are continuously rising (2). With the wide usage of the prostatespecific antigen (PSA)-based screening program, the majority of PCas is at a localized stage at diagnosis. Although patients with localized PCa can often be successfully treated with radical prostatectomy or radiotherapy, an increasing number of PCa patients may die of hormone refractory and metastatic disease. Carcinogenesis and the mechanisms influencing the progression and prognosis of $\mathrm{PCa}$ is a multistep process, which is likely a reflection of the underlying genomic diversity (3). Recent studies of PCa have indicated significant heterogeneity in the gene expression profiles, genetic and epigenetic alterations involving tumorigenesis and tumor progression of human PCa (4-6). Although genetic alterations are implicated in the activation or inactivation of cancer-associated genes, several types of epigenetic alterations have been identified in PCa, such as DNA methylation, loss of imprinting, and altered histone modification patterns (7-9).

DNA methylation is the most frequently studied epigenetic modification in PCa. It is involved not only in the initiation process of $\mathrm{PCa}$, but also in the progression phase of the disease (10). Since the high stability of DNA, the ease of analysis with the present techniques available and the ability 
to assess the biomarker in body fluids such as blood, urine and saliva, an increasing number of studies has focused on the identification of the DNA methylation-based biomarkers for PCa (11). With respect to DNA hypermethylation, this implies that several tumor-suppressor genes become hypermethylated during the initiation phase of $\mathrm{PCa}$ in a very consistent trend. In contrast, DNA hypomethylation, which is a decrease in the genomic DNA methylation level, is commonly linked to activation of oncogenes and chromosomal instability (12). Especially in PCa, recent studies have indicated that this particular epigenetic alteration may be associated with the advanced metastatic stage. Several genes, including those that encode glutathione S-transferase $\pi 1$ (GSTP1), adenomatous polyposis coli (APC), Ras association domain-containing protein 1 (RASSF1A) and prostaglandinendoperoxide synthase 2 (PTGS2), have been demonstrated to be hypermethylated in $\mathrm{PCa}$, but not in the normal prostate tissues $(6,13,14)$. Similarly, many hypomethylated genes have been identified and demonstrated to be associated with the recurrent status of PCa patients (15-17). These findings suggest that the DNA methylation of these loci may improve the diagnostic efficiency for PCa. Thus, it is reasonable to hypothesize that the investigation on the methylation status of certain genes is of great significance to elucidate the molecular mechanisms involved in the regulation of tumorigenesis and tumor progression in PCa.

Extracellular matrix metalloproteinase (MMP) inducer EMMPRIN (also known as CD147), a member of the immunoglobulin family, is a glycoprotein enriched on the surface of many types of tumor cells (18). CD147 has been demonstrated to be involved in tumor invasion and metastasis via stimulating MMPs synthesis, in neighboring fibroblasts and malignant cell proliferation via the activation of ERK1/2 and p38 mitogen-activated protein kinases, in enhancing angiogenesis via vascular endothelial growth factor, in inducing chemoresistant tumor cells via the production of hyaluronan, and in the resistance of cancer cells to anoikis through inhibition of Bim (19). The roles of CD147 and MMPs in tumor invasiveness have been confirmed immunohistochemically in several types of cancer cells. Especially in PCa, Wang et al (20) found that CD147 downregulation by RNAi technology could decrease the invasive capability of the PCa cells; data of our research group (21) and Madigan et al (22) both showed that CD147 could regulate MMPs and was involved in PCa progression. Hao et al (23) also reported that CD147 expression may be correlated with drug resistance during PCa metastasis and may be a useful potential therapeutic target in advanced disease; our research group further identified the potential role of CD147 as an independent predictor of biochemical recurrence, development of metastasis and reduced overall survival in PCa $(24,25)$. However, the mechanisms that result in the aberrant expression of CD147 in PCa are not fully elucidated. Thus, the aim of the present study was to investigate the CD147 promoter methylation status and the correlation with the tumorigenicity in PCa cells.

\section{Materials and methods}

Cell culture and epigenetic drug treatment. All the PCa cell lines (PC3, DU145, 22RV1 and Lncap) and the non-tumori- genic benign human prostatic epithelial cell lines (P69 and RWPE-1) were provided by Dr Chin-Lee Wu (Massachusetts General Hospital, Boston, MA, USA). The cells were grown in RPMI-1640 medium (Cellgro Mediatech, Inc., Manassas, VA, USA) with the necessary supplements. P69 and PC3 cell lines which were respectively treated with demethylating agent 5-aza-2'-deoxycytidine (Sigma-Aldrich Corp., St. Louis, MO, USA) were maintained in a RPMI-1640 medium with $1 \%$ fetal bovine serum, $1 \% 100 \mathrm{U} / \mathrm{ml}$ penicillin streptomycin mixture. P69 and PC3 cell lines were starved for $24 \mathrm{~h}$ before drug treatment. The cells were treated with 5-aza2 -deoxycytidine in different doses $(0,1,2,3,4$ and $5 \mu \mathrm{M})$ for 3 days at different time-points $(72,48,24,12,6$ and $0 \mathrm{~h}$ ), respectively. These concentrations are safe for cell culture without obvious toxicity. The growth medium was changed daily and the cells were harvested on day 4. All the experiments were performed in duplicate and repeated twice.

Clinical samples and tissue processing. The study was approved by the Research Ethics Committee of Guangzhou First People's Hospital, Guangzhou Medical College, China. An informed consent was obtained from all the patients. All the specimens were handled and made anonymous according to the ethical and legal standards.

Thirty-one PCa samples obtained from 31 PCa patients who underwent radical prostatectomy or transurethral resection of the prostate (TURP) at the Guangzhou First People's Hospital (Guangzhou, China) were collected. None of the patients recruited in this study received chemotherapy or radiotherapy prior to the surgery. In addition, 10 adjacent benign prostate tissues were collected as control tissue samples. All the tissues were frozen by liquid nitrogen and stored at $-80^{\circ} \mathrm{C}$. All the tissues were checked by H\&E staining before DNA/RNA extraction.

Real-time quantitative reverse transcriptase PCR. Total RNA was isolated from the cultured cells and the prostate tissues using TRIzol reagent (Invitrogen, Carlsbad, CA, USA) according to the manufacturer instructions. Reverse transcription was carried out using a ReverTra Ace reagent kit (Toyobo, Osaka, Japan). Regular PCR was used to amplify CD147 and glyceraldehyde-3-phosphate (GADPH) (internal control). In addition, real-time quantitative PCR was also performed with a MiniOpticon Real-Time PCR detection system (Bio-Rad, Hercules, CA, USA) in a SYBR-Green master mix (Takara, Otsu, Japan). The thermal cycling conditions comprised 1 cycle at $94^{\circ} \mathrm{C}$ for $4 \mathrm{~min}$, and 40 cycles at $94^{\circ} \mathrm{C}$ for $15 \mathrm{sec}, 60^{\circ} \mathrm{C}$ for $15 \mathrm{sec}$, and $72^{\circ} \mathrm{C}$ for $15 \mathrm{sec}$. All the data were analyzed using Opticon Monitor software (version 3.1; Bio-Rad) and the expression of CD147 was calculated as relative expression level to GAPDH using the comparative cycle threshold (CT) method. The sequences of PCR primers were forward, TGTACATTTTTAAAGGCAG AGATG and reverse, TTTCTTCCTTCCTGCTTTCAAA.

Western blot analysis. PCa cells and non-tumorigenic benign human prostatic epithelial cells were lysed in lysis buffer containing M-PER Mammalian Protein Extraction reagent and Protease Inhibitor Cocktail kit (both from Thermo Scientific, Rockford, IL, USA). Protein concentration of the cell lysates 
A

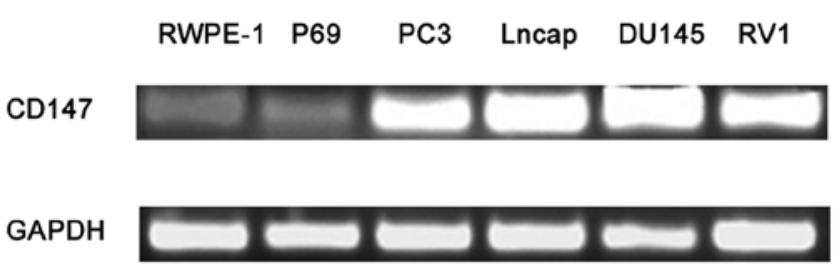

C

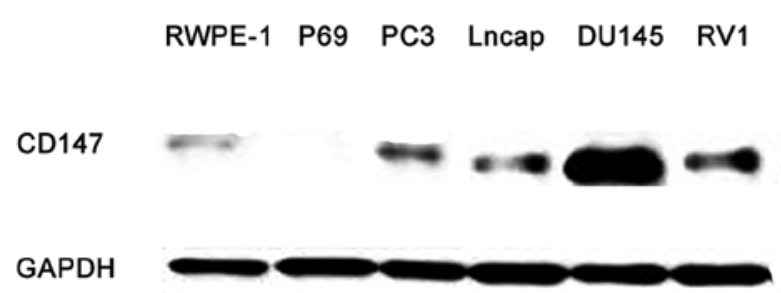

B

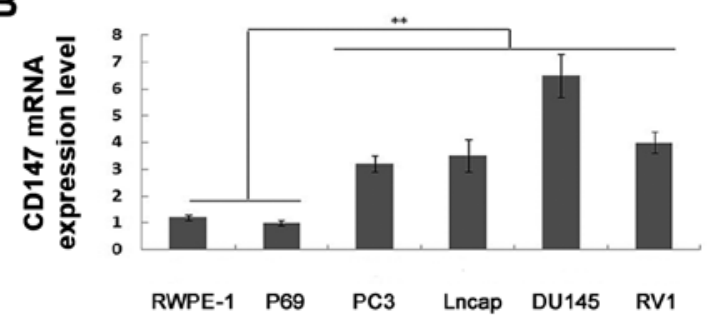

D

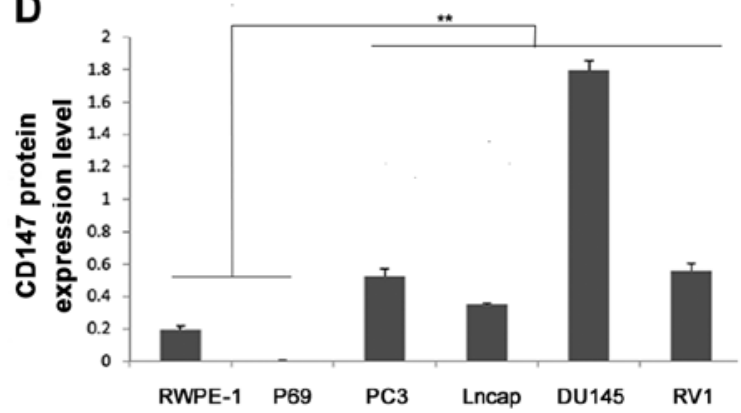

Figure 1. CD147 mRNA and protein expression in prostate cancer (PCa) and non-tumorigenic benign human prostatic epithelial cell lines. (A) The relative band quantification of real-time quantitative reverse transcriptase PCR for CD147 mRNA in various cell lines. (B) Statistical analysis of CD147 mRNA expression detected by real-time quantitative reverse transcriptase PCR analysis. (C) The relative blot of real-time western blot analysis for CD147 protein in various cell lines. (D) Statistical analysis of CD147 protein expression detected by western blot analysis. GAPDH was used as an internal control for 2 experiments, ${ }^{* *} \mathrm{P}<0.01$.

was determined by using the Synergy 2 Multi-Mode microplate reader (BioTek) according to the instructions of the manufacturer. Equal amounts of protein were loaded onto 4-20\% Tris-glycine gel (Invitrogen) following $130 \mathrm{v}$ for $90 \mathrm{~min}$. Then resolved proteins were electrophoretically transferred to the Immobilon-P transfer membranes (Millipore, Bedford, MA, USA). The membranes were blocked with $5 \%$ fetal bovine

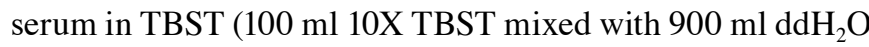
and $1 \mathrm{ml}$ Tween-20) for $1 \mathrm{~h}$ at room temperature followed by incubation with the anti-CD147 and anti-MMP2 rabbit monoclonal antibody (Abcam, Cambridge, MA, USA) at $4^{\circ} \mathrm{C}$ overnight. Blots were extensively washed with TBST 3 times and incubated with 1:3,000 dilution of goat against rabbit antibody diluted in TBST for $2 \mathrm{~h}$ at room temperature. Bound antibodies were detected with an enhanced chemiluminescence system (ECL, Amersham).

DNA extraction and sodium bisulfite genomic sequencing. Genomic DNA from cultured cells and prostate tissues were extracted with the QIAamp DNA mini kit (Qiagen, Valencia, CA, USA). All the genomic DNA was purified with the QIAquick PCR purification kit (Qiagen). Genomic DNA was treated with sodium bisulfite as described by EpiTect Bisulfite kit (Qiagen) as described by the instructions of the manufacturer. The sodium bisulfate-treated DNA was amplified by PyroMark PCR kit (Qiagen) for region (-940 to -670) of CD147 gene. The primers were (5'-TTT GGG TGA GTT GAG TTT AGT AGG-3') and (5'-TAC CAC AAA CTA CTA ACA TCA CTA AAC-3') designed using Pyrosequencing Assay Design software, version 1.0. The PCR conditions were as follows: $95^{\circ} \mathrm{C}$ for $15 \mathrm{~min}, 45$ cycles at $94^{\circ} \mathrm{C}$ for $45 \mathrm{sec}, 56^{\circ} \mathrm{C}$ for $30 \mathrm{sec}$, $72^{\circ} \mathrm{C}$ for $30 \mathrm{sec}$ and $72^{\circ} \mathrm{C}$ for $10 \mathrm{~min}$. The PCR products were then subcloned and sequenced (Life Technologies, Guangzhou, China).
Statistical analysis. SPSS version 13.0 for Windows (SPSS Inc., Chicago, IL, USA) and SAS 9.1 software (SAS Institute, Cary, NC, USA) was used for statistical analysis. All the results were confirmed in at least three separate experiments and expressed as mean \pm SD. Data were analyzed for statistical significance by the Student's t-test or the independent samples t-test. The Spearman correlation was calculated between methylation intensity and mRNA levels of CD147. Differences were considered to be statistically significant when the P-value was $<0.05$.

\section{Results}

Overexpression of CD147 in PCa cell lines. Real-time quantitative reverse transcriptase PCR and western blot analyses were, respectively, performed to detect the expression of CD147 at mRNA and protein levels in 4 PCa and 2 nontumorigenic benign human prostatic epithelial cell lines. As shown in Fig. 1, both CD147 mRNA and protein were highly expressed in PCa cell lines compared to non-tumorigenic benign human prostatic epithelial cell lines (all $\mathrm{P}<0.01$ ), which were consistent with our previous data in human $\mathrm{PCa}$ and non-cancerous prostate tissues $(21,24,25)$.

DNA promoter hypomethylation of CD147 in PCa cell lines. To evaluate whether CD147 promoter region contains an area rich with $\mathrm{CpG}$ dinucleotides, we used the EMBOSS CpG plot (EMBL-EBI: http://www.ebi.ac.uk/Tools/emboss/cpgplot/index. html). The 'cpgplot' identifies the $\mathrm{CpG}$ islands in one or more nucleotide sequences. The ratio of the observed to the expected number of GC dinucleotide patterns was calculated over a sequence region. The calculated ratios were plotted graphically, together with the regions matching the definition of the program 'CpG island' (a CG dinucleotide rich area). An area 
A
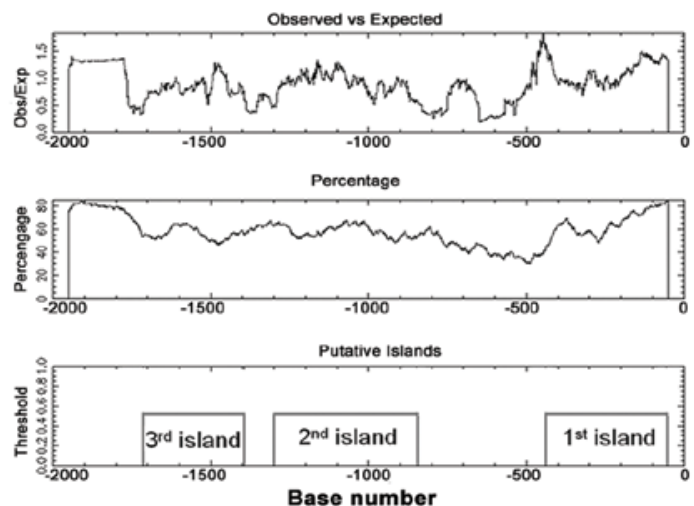

C F' $^{\prime}$
TTGGGTITTIAGTTGAGTTTIAGTAA
TAAACCCAAACTCTCAACA

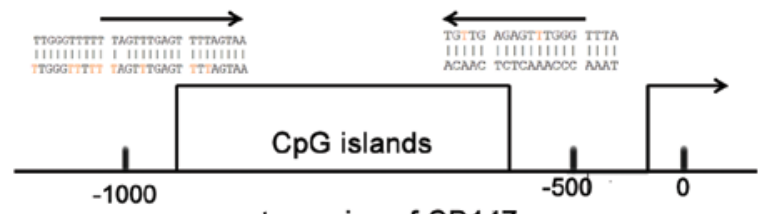

B

12

TAAACAAGTTTCCCGCCGGTCCTGATCTGCAGAGATGGCACCTGCCACO $\begin{array}{llll}3 & 4 & 5 & 6\end{array}$ CACTCTGCACATCCCCCTCCCGTCGCCACGGGTCTTCCCGCCAGTGTA $\begin{array}{lll}7 & 8 & 9\end{array}$ GCCACATTCCTGCCCCTITCAGTAGCCCTCGCGTCGGCTIAGTCTG $10 \quad 11 \quad 12$ CGGTCCTCTGCATTGCGACTCCGAGTTAACTCCAACACACACTICA 13 14 14 ACCTCCAAGAGACGCCCCCACCTGTGTCGCCCCAATAGCGACTTITCTC $16 \quad 17 \quad 1819$

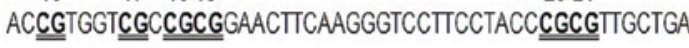

GAGTCTGGGT Transcription Starting Site

D 123456789101112131415161718192021

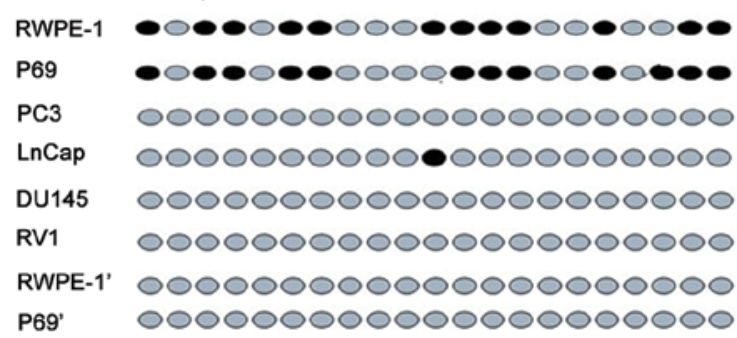

Figure 2. DNA methylation profile of the $\mathrm{CpG}$ island at the CD147 promoter region. (A) Map of predicted CpG islands. Location of three CpG islands were predicted in the CD147 promoter. The GC percentage and the observed/expected CpG were calculated using the EMBOSS CpG plot program. (B) Schematic representation of the $\mathrm{CpG}$ island. The $21 \mathrm{CG}$ dinucleotides are denoted with grey shading. $\mathrm{CpG}$ sites were numbered from 1 to 21 relative to the transcription start site (C) The 2nd CpG island in the promoter region of the CD147 gene. Forward (F') and reverse ( $\left.\mathrm{R}^{\prime}\right)$ primers for the bisulfite genomic sequence are shown. (D) Sequences of the CD147 promoter region after the bisulfite modification were analyzed in the prostate cancer (PCa), non-tumorigenic benign human prostatic epithelial and benign prostatic cell lines treated with 5-aza-2'-deoxycytidine. Black circle, methylated cytosine; grey circle, unmethylated cytosine in the dinucleotide $\mathrm{CpG}$.

was considered a $\mathrm{CpG}$ island if an average of 10 nucleotide sequences which were at least 200 bases, contained over $50 \%$ $\mathrm{G}$ and $\mathrm{C}$ nucleotides, and the calculated observed/expected ratio was over 0.6. We analyzed the DNA sequence of the CD147 promoter region (NG_007468.1) using the EMBOSS tool, the result of evaluation for the $\mathrm{CpG}$ islands is shown in Fig. 2A, where $3 \mathrm{CpG}$ islands are predicted. Among them, the sequence of -500 has been reported by Sharma et al (5). According to the results of Liang et al (26), a fragment -1024 to -59 bp upstream of the CD147 coding region was sufficient to promote transcription. In the present study, we chose $1-2 \mathrm{CpG}$ islands located in this fragment to further study. To investigate the correlation between DNA methylation status and CD147 expression level, promoter methylation was analyzed for CD147 by genomic bisulfite sequencing in 4 PCa (PC3, Lncap, DU145 and RV1) and 2 non-tumorigenic benign human prostatic epithelial cell lines (RWPE-1 and P69). However, our data showed that no methylated $\mathrm{CpG}$ spot in typical $46 \mathrm{CpG}$ sites was detected in this region of the 1st $\mathrm{CpG}$ island in either PCa or non-tumorigenic benign human prostatic epithelial cells. As shown in Fig. 2B and C, a total of $21 \mathrm{CpG}$ sites in the $2 \mathrm{nd} \mathrm{CpG}$ island were assessed by PCR and sequencing. The non-tumorigenic benign human prostatic epithelial cell lines exhibited a denser methylation pattern at the CD147 promoter region than the corresponding PCa cell lines (Fig. 2D), suggesting that the hypomethylation of the CD147 promoter region may contribute to the increased CD147 expression in the PCa cells. We also found that the non-tumorigenic benign human prostatic epithelial cell lines treated with 5-aza-2'-deoxycytidine may exhibit the same methylation pattern as the PCa cell lines (Fig. 2D).
Re-expression of CD147 induced by 5-aza-2'-deoxycytidine treatment in non-tumorigenic benign human prostatic epithelial cell line. To examine whether the CD147 expression in the non-tumorigenic benign human prostatic epithelial cell line P69 could be restored by the treatment with DNA methylation inhibitors, we treated the P69 cells with 5-aza-2'-deoxycytidine and then analyzed for CD147 protein expression by western blot analysis. As shown in Fig. 3A and B, the treatment of 5-aza-2'-deoxycytidine is able to induce CD147 expression in P69 cells in a dose- and time-dependent manner. Since MMP-2 has been demonstrated to be one of the most important downstream targets of CD147, we further examined whether MMP-2 expression in P69 cells could be activated by the treatment with DNA methylation inhibitors. As shown in Fig. 3C and D, the treatment of 5-aza-2'-deoxycytidine is also able to activate MMP-2 expression in P69 cells in a dose- and time-dependent manner.

Hypomethylation affects CD147 expression in clinical PCa tissues and is related to the metastasis of cancer. To investigate the epigenetic mechanism responsible for CD147 transcription in human PCa, the DNA methylation status of the CD147 promoter region (2nd CpG islands) was determined in $\mathrm{PCa}$ and corresponding non-cancerous prostate tissues by sodium bisulfite genomic sequencing and the expression levels of CD147 mRNA were also detected by real-time quantitative RT-PCR using the same clinical samples. As shown in Fig. 4A and C, the $\mathrm{CpG}$ sites in the island exhibited significant hypomethylation in $\mathrm{PCa}$ tissues compared to corresponding benign prostate tissues (ratio of methylation PCa vs. benign: 0.24 vs. 0.64 , 
A

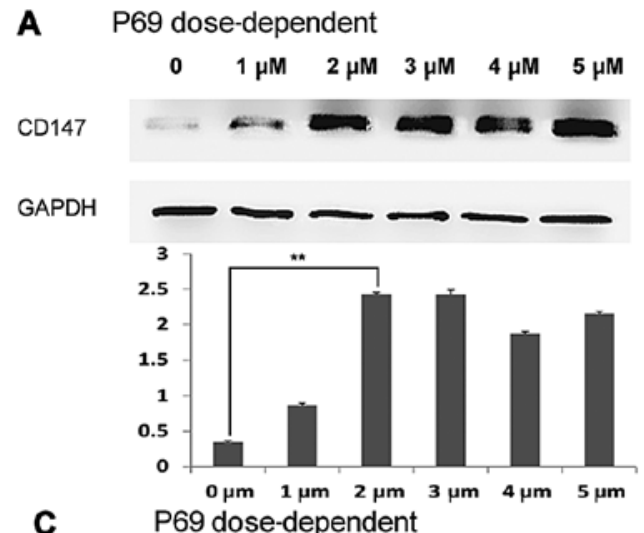

MMP2

GAPDH
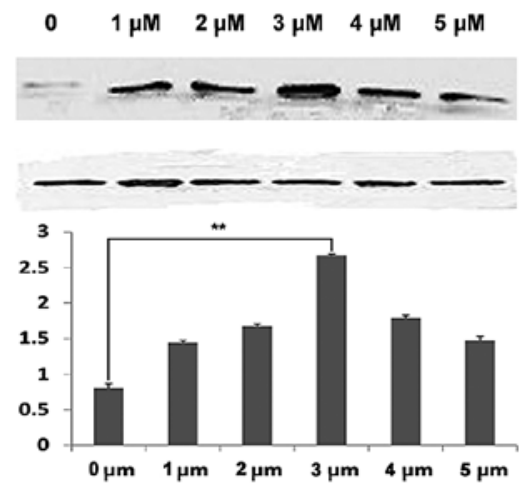

B

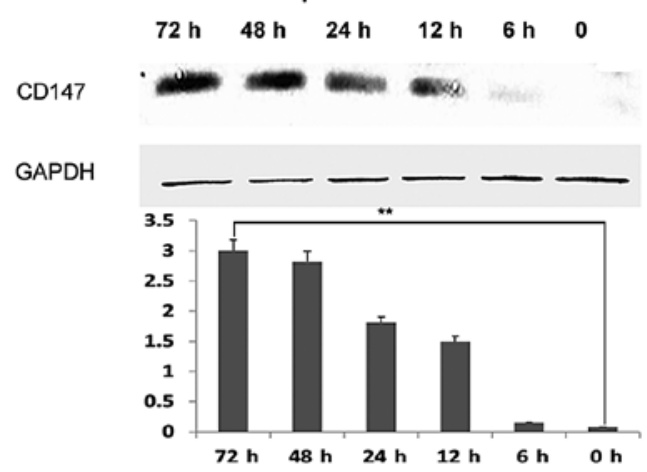

D

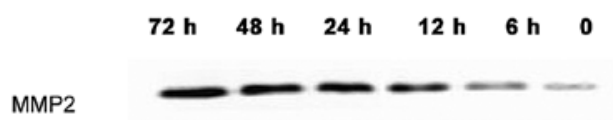

GAPDH
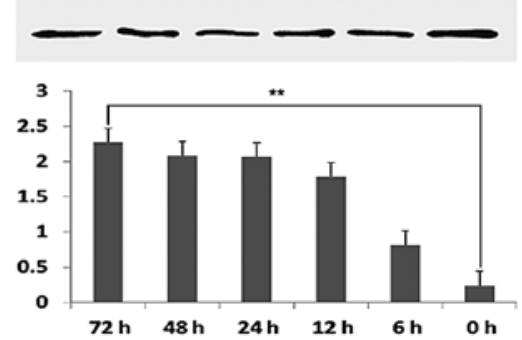

Figure 3. Re-expression of CD147 induced by 5-aza-2'-deoxycytidine treatment in non-tumorigenic benign human prostatic epithelial cell line. (A and B) The treatment with 5-aza-2'-deoxycytidine is able to induce CD147 expression in P69 cells in a dose- and time-dependent manner. (C and D) The 5-aza-2'-deoxycytidine treatment is able to activate matrix metalloproteinase (MMP)-2 expression in P69 cells in a dose- and time-dependent manner, ${ }^{* *} \mathrm{P}<0.01$.

$\mathrm{P}<0.01)$. Importantly, $\mathrm{CpG}$ sites in the island exhibited significant hypomethylation in metastatic PCa tissues compared to non-metastatic PCa tissues (ratio of methylation metastatic PCa vs. non-metastatic PCa: 0.10 vs. 0.32, $\mathrm{P}=0.009$, Fig. 4D). Similarly, the expression levels of CD147 mRNA in PCa tissues were significantly higher than those in corresponding benign prostate tissues (PCa vs. benign: 3.12 vs. 1.18, $\mathrm{P}<0.01$, Fig. 4B) and the expression levels of CD147 mRNA in metastatic PCa tissues were also significantly higher than those in non-metastatic $\mathrm{PCa}$ tissues (PCa vs. benign: 4.30 vs. 2.55 , $\mathrm{P}=0.046$, Fig. 4E). In addition, methylation intensity was significantly correlated inversely with CD147 mRNA levels in PCa tissues (Fig. 4B). CD147 mRNA levels in PCa tissues with DNA hypomethylation were significantly higher than those with DNA methylation (methylated PCa vs. unmethylated PCa: 2.12 vs. $3.83, \mathrm{P}=0.017$ ), but no statistical difference was found between mythylated and hypomethylated benign prostate tissues ( $\mathrm{P}>0.05$, Fig. 4F). There was a significant negative correlation between CD147 mRNA levels and the number of methylated sites in PCa tissues (Pearson correlation $\mathrm{r}=-0.467$, $\mathrm{P}=0.008$, Fig. $4 \mathrm{G})$.

\section{Discussion}

Many studies have emphasized that epigenetic alterations play an important role in the initiation of various human cancers. Aberrant expression of cancer-related genes may benefit the expansion of the cells in the early abnormal cloning and 'addict' cancer cells to the subsequent genetic and epigenetic alterations that further promote tumor progression (27). According to our previous data, as well as that of other research groups, CD147 functions as an oncogene and is a good target for diagnosis, prediction for disease progression and therapy of PCa (20-25). In the present study, we observed the overexpression of CD147 mRNA and protein in several PCa cell lines, consistent with our previous studies on PCa tissues $(21,24,25)$. Notably, we found that overexpression was associated with hypomethylation of CD147 promoter in PCa cell lines. In addition, re-expression of CD147 and its downstream target MMP-2 are able to be induced by demethylation agent 5-aza-2'-deoxycytidine in lowexpressing non-tumorigenic benign human prostatic epithelial cell lines. Furthermore, PCa tissues displayed decreased DNA methylation in the promoter region of CD147 compared to corresponding non-cancerous prostate tissues, and methylation intensity correlated inversely with CD147 mRNA levels. There was a significantly negative correlation between CD147 mRNA levels and the number of methylated sites in the PCa tissues. Although still leaving much to investigate, to our knowledge, this is the first attempt to explore a possible mechanism leading to the abnormal CD147 expression levels in PCa.

Despite the comprehensive investigation of CD147 expression by previous studies (20-25), the regulatory mechanisms underlying aberrant CD147 expression in various human cancers are not yet fully elucidated. Since the aberrant methylation of promoter $\mathrm{CpG}$ islands has been widely considered as a kind of regulatory mechanism of silencing tumor suppressors or activating oncogenes, it is reasonable to assume the possible role of aberrant methylation on CD147 expression levels. To validate 


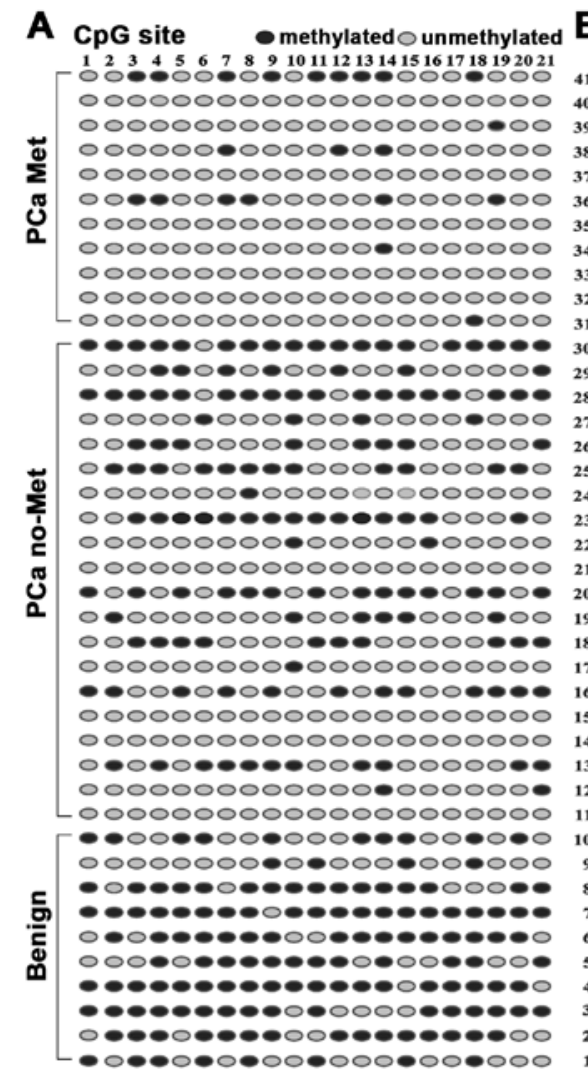

CD147 mRNA expression level
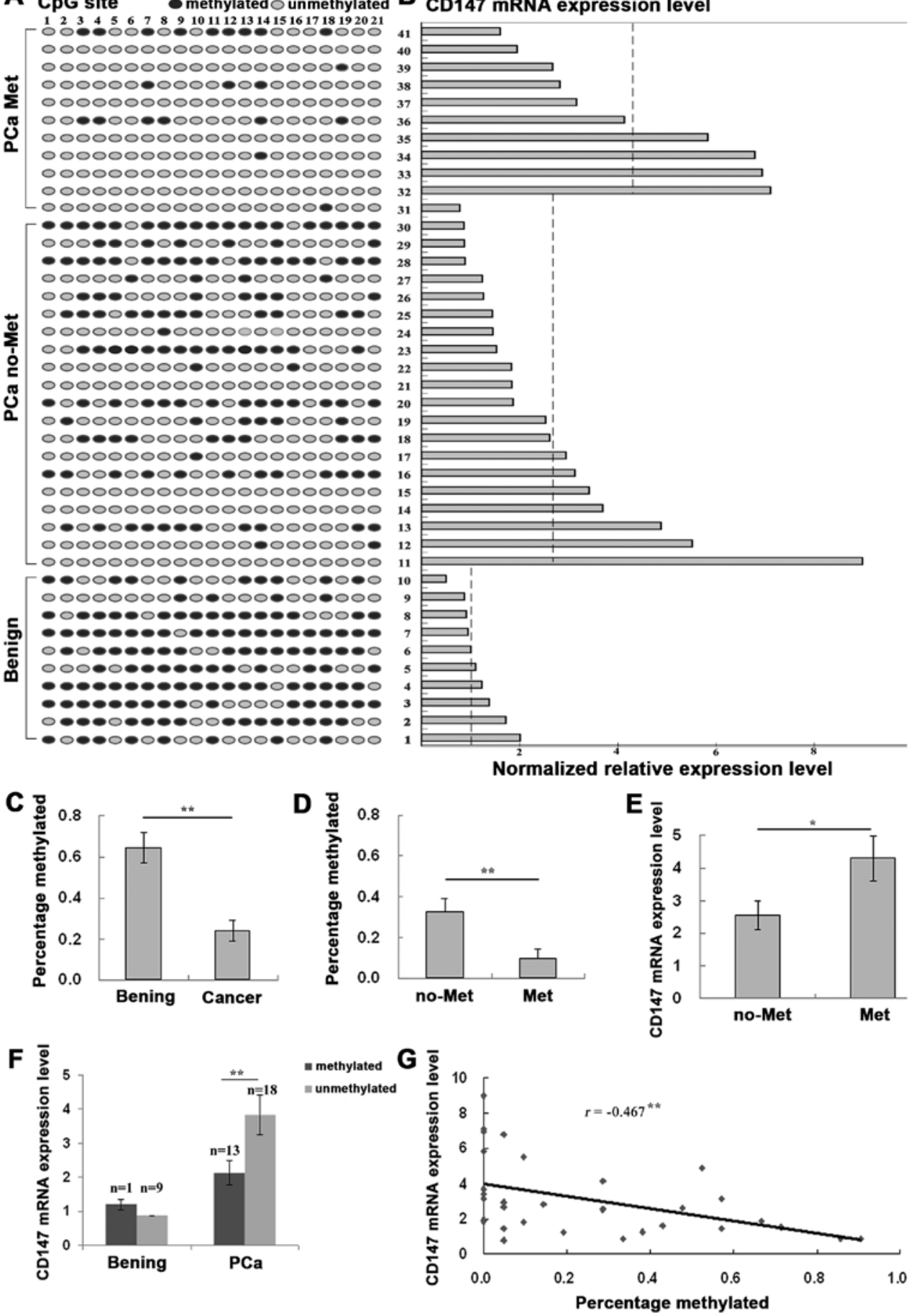

Figure 4. Hypomethylation in the promoter of CD147 in human prostate cancer (PCa) tissues. (A) Methylation patterns in the promoter region of CD147 in clinical PCa tissues. Closed circles, methylation; open circles, unmethylation. Ten individual clones were sequenced for each sample. (B) mRNA expression of CD147 was detected by RT-qPCR for the same samples. (C and D) Methylation intensity (percent of methylated CpG site) between benign and cancer tissues, and between cancer tissues with clinical non-metastasis and metastasis. (E) mRNA expression of CD147 in cancer tissues with clinical non-metastasis and metastasis. (F) mRNA expression of CD147 in benign and PCa tissues with different mythylated status groups. (G) Correlation between the relative CD147 mRNA levels and CD147 promoter methylation intensity (percent of methylated CpG site) in PCa tissues. Bar graphs show mean $\pm \mathrm{SE} .{ }^{*} \mathrm{P}<0.05,{ }^{* *} \mathrm{P}<0.01$.

this hypothesis, we firstly investigated the methylation status of CD147 promoter in PCa and non-tumorigenic benign human prostatic epithelial cell lines. The results showed a significantly lower level of methylation status of CD147 promoter in the PCa cells when compared with the benign controls. There was a correlation between the promoter hypomethylation and the CD147 overexpression, suggesting that the promoter demethylation increased the expression of CD147 in the PCa cells, which was further validated by our subsequent experiments in cell lines P69 and PC3 treated with the demethylating agent. Then, the present study also showed that human PCa tissues displayed a relatively hypomethylated CD147 promoter, compared to corresponding non-cancerous prostate tissues. The above findings confirm our initial hypothesis that DNA methylation may play a crucial role in regulating the expression of CD147 in PCa. 
In conclusion, our data offer convincing evidence for the first time that DNA promoter hypomethylation of CD147 may be one of the regulatory mechanisms involved in the cancer-related overexpression of CD147 and may play a crucial role in the tumorigenesis of human $\mathrm{PCa}$.

\section{Acknowledgements}

This study was supported by grants from the National Natural Science Foundation of China (nos. 81200550 and 81170699), the Medical Research Fund of Guangdong Province (no. A2012489) and the Science and Technology Project of Bureau of Health in Guangzhou Municipality (no. 20121A011004).

\section{References}

1. Siegel R, Naishadham D and Jemal A: Cancer statistics, 2013. CA Cancer J Clin 63: 11-30, 2013.

2. Molitierno J, Evans A, Mohler JL, Wallen E, Moore D and Pruthi RS: Characterization of biochemical recurrence after radical prostatectomy. Urol Int 77: 130-134, 2006.

3. Klotz L: Hormone therapy for patients with prostate carcinoma. Cancer 88 (Suppl 12): 3009-3014, 2000.

4. Schoenborn JR, Nelson P and Fang M: Genomic profiling defines subtypes of prostate cancer with the potential for therapeutic stratification. Clin Cancer Res 19: 4058-4066, 2013.

5. Sharma $\mathrm{S}$ and Watabe K: Biomarkers and mechanisms associated with recurrent prostate cancer. Front Biosci (Landmark Ed) 19: 339-351, 2014.

6. Devaney JM, Wang S, Funda S, Long J, Taghipour DJ, Tbaishat R, Furbert-Harris P, Ittmann $M$ and Kwabi-Addo B: Identification of novel DNA-methylated genes that correlate with human prostate cancer and high-grade prostatic intraepithelial neoplasia. Prostate Cancer Prostatic Dis 16: 292-300, 2013.

7. Yang B, Bhusari S, Kueck J, Weeratunga P, Wagner J, Leverson G, Huang W and Jarrard DF: Methylation profiling defines an extensive field defect in histologically normal prostate tissues associated with prostate cancer. Neoplasia 15: 399-408, 2013.

8. Lin PC, Giannopoulou EG, Park K, Mosquera JM, Sboner A, Tewari AK, Garraway LA, Beltran H, Rubin MA and Elemento O: Epigenomic alterations in localized and advanced prostate cancer. Neoplasia 15: 373-383, 2013.

9. Aryee MJ, Liu W, Engelmann JC, Nuhn P, Gurel M, Haffner MC Esopi D, Irizarry RA, Getzenberg RH, Nelson WG, et al: DNA methylation alterations exhibit intraindividual stability and interindividual heterogeneity in prostate cancer metastases. Sci Transl Med 5: 169ra10, 2013

10. Majumdar S, Buckles E, Estrada J and Koochekpour S: Aberrant DNA methylation and prostate cancer. Curr Genomics 12: 486-505, 2011.

11. Dimitriadis E, Kalogeropoulos T, Velaeti S, Sotiriou S, Vassiliou E, Fasoulis L, Klapsas V, Synesiou M, Apostolaki A, Trangas T, et al: Study of genetic and epigenetic alterations in urine samples as diagnostic markers for prostate cancer. Anticancer Res 33: 191-197, 2013.

12. Yu YP, Ding Y, Chen R, Liao SG, Ren BG, Michalopoulos A, Michalopoulos G, Nelson J, Tseng GC and Luo JH: Whole-genome methylation sequencing reveals distinct impact of differential methylations on gene transcription in prostate cancer. Am J Pathol 183: 1960-1970, 2013.
13. Haldrup C, Mundbjerg K, Vestergaard EM, Lamy P, Wild P, Schulz WA, Arsov C, Visakorpi T, Borre M, Høyer S, et al: DNA methylation signatures for prediction of biochemical recurrence after radical prostatectomy of clinically localized prostate cancer. J Clin Oncol 31: 3250-3258, 2013.

14. Chao C, Chi M, Preciado M and Black MH: Methylation markers for prostate cancer prognosis: A systematic review. Cancer Causes Control 24: 1615-1641, 2013.

15. Dietrich D, Hasinger O, Bañez LL, Sun L, van Leenders GJ, Wheeler TM, Bangma CH, Wernert N, Perner S, Freedland SJ, et al: Development and clinical validation of a real-time PCR assay for PITX2 DNA methylation to predict prostate-specific antigen recurrence in prostate cancer patients following radical prostatectomy. J Mol Diagn 15: 270-279, 2013.

16. Kron K, Trudel D, Pethe V, Briollais L, Fleshner N, van der Kwast T and Bapat B: Altered DNA methylation landscapes of polycomb-repressed loci are associated with prostate cancer progression and ERG oncogene expression in prostate cancer. Clin Cancer Res 19: 3450-3461, 2013.

17. Vasiljević N, Ahmad AS, Beesley C, Thorat MA, Fisher G, Berney DM, Møller H, Yu Y, Lu YJ, Cuzick J, et al: Association between DNA methylation of HSPB1 and death in low Gleason score prostate cancer. Prostate Cancer Prostatic Dis 16: 35-40, 2013.

18. Gabison EE, Mourah S, Steinfels E, Yan L, Hoang-Xuan T, Watsky MA, De Wever B, Calvo F, Mauviel A and Menashi S: Differential expression of extracellular matrix metalloproteinase inducer (CD147) in normal and ulcerated corneas: role in epitheliostromal interactions and matrix metalloproteinase induction. Am J Pathol 166: 209-219, 2005.

19. Sun J and Hemler ME: Regulation of MMP-1 and MMP-2 production through CD147/extracellular matrix metalloproteinase inducer interactions. Cancer Res 61: 2276-2281, 2001.

20. Wang L, Wu G, Yu L, Yuan J, Fang F, Zhai Z, Wang F and Wang H: Inhibition of CD147 expression reduces tumor cell invasion in human prostate cancer cell line via RNA interference. Cancer Biol Ther 5: 608-614, 2006.

21. Zhong WD, Han ZD, He HC, Bi XC, Dai QS, Zhu G, Ye YK, Liang YX, Qin WJ, Zhang Z, et al: CD147, MMP-1, MMP-2 and MMP-9 protein expression as significant prognostic factors in human prostate cancer. Oncology 75: 230-236, 2008.

22. Madigan MC, Kingsley EA, Cozzi PJ, Delprado WJ, Russell PJ and $\mathrm{Li} \mathrm{Y}$ : The role of extracellular matrix metalloproteinase inducer protein in prostate cancer progression. Cancer Immunol Immunother 57: 1367-1379, 2008.

23. Hao JL,Cozzi PJ,Khatri A,Power CA and Li Y: CD147/EMMPRIN and CD44 are potential therapeutic targets for metastatic prostate cancer. Curr Cancer Drug Targets 10: 287-306, 2010.

24. Han ZD, Bi XC, Qin WJ, He HC, Dai QS, Zou J, Ye YK, Liang YX, Zeng GH, Chen ZN, et al: CD147 expression indicates unfavourable prognosis in prostate cancer. Pathol Oncol Res 15: 369-374, 2009.

25. Zhong WD, Liang YX, Lin SX, Li L, He HC, Bi XC, Han ZD, Dai QS, Ye YK, Chen QB, et al: Expression of CD147 is associated with prostate cancer progression. Int J Cancer 130: 300-308, 2012.

26. Liang L, Major T and Bocan T: Characterization of the promoter of human extracellular matrix metalloproteinase inducer (EMMPRIN). Gene 282: 75-86, 2002.

27. Truong M, Yang B, Livermore A, Wagner J, Weeratunga $P$, Huang W, Dhir R, Nelson J, Lin DW and Jarrard DF: Using the epigenetic field defect to detect prostate cancer in biopsy negative patients. J Urol 189: 2335-2341, 2013. 